\title{
NONCLASSICAL SIMPLE LIE ALGEBRAS ${ }^{1}$
}

\author{
BY ROBERT LEE WILSON
}

Communicated by Louis Auslander, April 1, 1969

Introduction. Let $\Phi$ be an algebraically closed field of characteristic $p>3$. In addition to the finite dimensional classical simple Lie algebras [12] over $\Phi$ a number of families of finite dimensional nonclassical simple Lie algebras over $\Phi$ have been discovered [1]-[3], [5]-[9], [13]. Until recently no general connection has been known between these algebras and any family of Lie algebras over fields of characteristic 0 .

Recently Kostrikin and Shafarevitch [11] have given a unified construction of all known finite dimensional nonclassical simple restricted Lie algebras over $\Phi$. These algebras are obtained as the analogues in prime characteristic of the simple infinite Lie algebras of Cartan type over $C$.

We give here a generalization of the Kostrikin-Shafarevitch construction which gives all known finite dimensional nonclassical simple (not necessarily restricted) Lie algebras over $\Phi$, as well as some which are new. ${ }^{2}$

I. Definition of Lie algebras of Cartan type. The infinite Lie algebras of Cartan type are certain Lie algebras over $C$ which arise in the study of pseudogroups [10], [15]. They are characterized by the following conditions:

(1) $L$ has a decreasing filtration $L=L_{-1} \supset L_{0} \supset L_{1} \supset \cdots$.

(2) $\cap L_{i}=(0)$.

(3) $\left[L_{i}, L_{j}\right] \subseteq L_{i+j}$ for $-1 \leqq i, j$ (where $L_{-2}=L$ ).

(4) If $x \in L_{i}$ and $x \notin L_{i+1}$ for some $i \geqq 0$ then there exists $y \in L$ such that $[x y] \notin L_{i}$.

(5) $\operatorname{dim} L_{-1} / L_{0}<\infty$.

(6) $\operatorname{dim} L=\infty$.

1 These results are contained in the author's doctoral dissertation written under the guidance of Professor G. B. Seligman at Yale University. The author was a National Science Foundation Graduate Fellow at Yale.

2 Added in proof. In a recent paper (Graded Lie algebras of finite characteristic, Izv. Akad. Nauk SSSR Ser. Mat. 30 (1969), 251-322) Kostrikin and Shafarevitch have also studied the nonrestricted case and have obtained results which substantially overlap those of this paper. 
The simple infinite Lie algebras of Cartan type over $C$ have been determined [4], [10], [15]. They are:

$$
\begin{aligned}
& w(m)=\operatorname{Der} C\left[\left[x_{1}, \cdots, x_{m}\right]\right], \quad m \geqq 1 . \\
& \mathcal{S}(m)=\left\{D \in W(m) \mid D \omega=0, \omega=d x_{1} \wedge \cdots \wedge d x_{m}\right\}, \quad m \geqq 2 . \\
& \mathcal{V}(2 r)=\left\{D \in W(2 r) \mid D \omega=0, \omega=\sum_{i=1}^{r} d x_{i} \wedge d x_{i+r}\right\}, \quad r \geqq 2 \text {. } \\
& R(2 r+1)=\left\{D \in W(2 r+1) \mid D \omega=u \omega, u \in C\left[\left[x_{1}, \cdots, x_{2 r+1}\right]\right],\right. \\
& \left.\omega=d x_{2 r+1}+\sum_{i=1}^{r} x_{i} d x_{i+r}-x_{i+r} d x_{i}\right\}, \quad r \geqq 1 .
\end{aligned}
$$

(The action of a derivation $D$ on the algebra $D$ of differential forms is that of the Lie derivative $[16$, p. 92]. Thus $D$ is the exterior algebra on $\left\{d x_{1}, \cdots, d x_{m}\right\}$ over $\mathbf{C}\left[\left[x_{1}, \cdots, x_{m}\right]\right], d f=\sum\left(\partial f / \partial x_{i}\right) d x_{i}, D(d f)$ $=d(D f), D(f \omega)=(D f) \omega+f(D \omega)$, and $D(\omega \wedge \eta)=D \omega \wedge \eta+\omega \wedge D \eta$ for all $f \in C\left[\left[x_{1}, \cdots, x_{m}\right]\right]$ and all $\omega$ and $\eta \in D$.)

We now consider certain $\boldsymbol{Z}$-subalgebras of these algebras. Define $A(m)=\{\alpha:\{1, \cdots, m\} \rightarrow Z \mid \alpha(i) \geqq 0$ for $1 \leqq i \leqq m\}$. Define $\epsilon_{i} \in A(m)$ by $\epsilon_{i}(j)=\delta_{i j}$. For $\alpha, \beta \in A(m)$ define

$$
\alpha !=\Pi \alpha(i) !, \quad\left(\begin{array}{l}
\alpha \\
\beta
\end{array}\right)=\Pi\left(\begin{array}{c}
\alpha(i) \\
\beta(i)
\end{array}\right)
$$

and

$$
|\alpha|=\sum \alpha(i) .
$$

Let $Q(m)=C\left[\left[x_{1}, \cdots, x_{m}\right]\right]$. For $\alpha \in A(m)$ define $x^{\alpha}=\prod x^{\alpha(i)} / \alpha(i)$ ! $\in Q(m)$. Set $\bar{Q}(m)=\left\{\sum a_{\alpha} x^{\alpha} \mid a_{\alpha} \in \boldsymbol{Z}\right\} \subset a(m)$ where the summation extends over all $\alpha \in A(m)$ and infinite sums are allowed. Then $\bar{Q}(m)$ is a $\boldsymbol{Z}$-subalgebra of $\boldsymbol{Q}(m)$. Set $\overline{\mathscr{W}}(m)=$ Der $\bar{Q}(m)$ $=\left\{\sum f_{i}\left(\partial / \partial x_{i}\right) \mid f_{i} \in \bar{Q}(m), 1 \leqq i \leqq m\right\}$. Then $\overline{\mathscr{W}}(m)$ is a $Z$-subalgebra of $\mathscr{W}(m)$. Now let $\Phi$ be an arbitrary field and define $\mathfrak{A}(m)=\bar{a}(m) \otimes_{z} \Phi$ and $W(m)=\overline{\mathscr{W}}(m) \otimes_{z} \Phi$. Then $\mathfrak{A}(m)$ is an associative algebra over $\Phi$ with multiplication defined by bilinearity and

$$
x^{\alpha} x^{\beta}=\left(\begin{array}{c}
\alpha+\beta \\
\alpha
\end{array}\right) x^{\alpha+\beta}
$$

and $W(m)=\left\{\sum f_{i} D_{i} \mid f_{i} \in \mathfrak{A}(m)\right\}$ (where $D_{i}$ is the image of $\left.\partial / \partial x_{i}\right)$ is a Lie algebra of derivations of $\mathfrak{A}(m)$. The action of $W(m)$ on $\mathfrak{A}(m)$ is 
given by

$$
D_{i} x^{\alpha}=x^{\alpha-\epsilon_{i}}
$$

and multiplication in $W(m)$ is given by

$$
\left[x^{\alpha} D_{i}, x^{\beta} D_{j}\right]=\left(\begin{array}{c}
\alpha+\beta-\epsilon_{i} \\
\alpha
\end{array}\right) x^{\alpha+\beta-\epsilon_{i}} D_{j}-\left(\begin{array}{c}
\alpha+\beta-\epsilon_{j} \\
\beta
\end{array}\right) x^{\alpha+\beta-\epsilon_{i}} D_{i} .
$$

Define $S(m)=S(m) \cap \bar{W}(m)$ and $S(m)=\overline{\mathcal{S}}(m) \otimes_{z} \Phi$. Define $V(2 r)$ and $R(2 r+1)$ similarly. Now $W(m), S(m), V(2 r)$, and $R(2 r+1)$ are infinite dimensional Lie algebras over $\Phi$. We now consider certain finite dimensional subalgebras of them.

Define $A\left(n_{1}, \cdots, n_{m}\right)=\left\{\alpha \in A(m) \mid \alpha(i)<p^{n_{i}}, 1 \leqq i \leqq m\right\} \quad$ (where characteristic $\Phi=p)$. Then $\mathfrak{A}\left(n_{1}, \cdots, n_{m}\right)=\left\langle x^{\alpha} \mid \alpha \in A\left(n_{1}, \cdots, n_{m}\right)\right\rangle$ is a subalgebra of $\mathfrak{A}(m)$. Hence $W\left(n_{1}, \cdots, n_{m}\right)=$ the stabilizer in $W(m)$ of $\mathfrak{A}\left(n_{1}, \cdots, n_{m}\right)$ is a subalgebra of $W(m)$.

Let $\mathfrak{P}(m)_{i}=\left\{\sum a_{\alpha} x^{\alpha} \mid a_{\alpha}=0\right.$ unless $\left.|\alpha| \geqq i+1\right\}$. Then $\mathfrak{A}(m)$ is a topological algebra with topology defined by taking $\left\{\mathfrak{A}(m)_{i} \mid i \geqq 0\right\}$ to be a base of neighborhoods of 0 . For $1 \leqq i \leqq r$ define $\mathfrak{A}_{i}(2 r)$ $=\left\{\sum a_{\alpha} x^{\alpha} \mid a_{\alpha}=0\right.$ if $\alpha(j) \neq 0$ for some $j \neq i$ or $\left.i+r\right\}$.

Let $\phi$ be an automorphism of $\mathfrak{A}(m)$. If $D \in W(m)$ then $D^{\phi}=\phi D \phi^{-1}$ is a derivation of $\mathfrak{X}(m)$. Following Ree [13] we say that $\phi$ is an admissible automorphism of $\mathfrak{A}(m)$ (with respect to $W(m)$ ) if $\phi$ is continuous and $W(m)^{\phi} \subseteq W(m)$.

LEMMA 1. If $\phi$ is an admissible automorphism of $\mathfrak{A}(m)$ then $\operatorname{det}\left(D_{i} \phi x^{\epsilon_{j}}\right)$ is a unit in $\mathfrak{U}(m)$.

Definition 1. A Lie algebra $L$ over a field $\Phi$ of characteristic $p>0$ is a Lie algebra of Cartan type if $L=K^{\prime \prime}$ where $K$ is one of the following algebras:

(7) $W\left(n_{1}, \cdots, n_{m}\right)$ where $\sum\left(p^{n_{i}}-1\right)>2$.

(8) $S(m) \phi W\left(n_{1}, \cdots, n_{m}\right)$ where $m \geqq 2, \sum\left(p^{n_{i}}-1\right)>3, \phi$ is an admissible automorphism of $\mathfrak{A}(m)$ and $a^{-1} D_{i} a \in \mathfrak{U}\left(n_{1}, \cdots, n_{m}\right)$ for $1 \leqq i \leqq m$ where $a=\operatorname{det}\left(D_{i} \phi x^{\epsilon_{i}}\right)$.

(9) $V(2 r) \phi W\left(n_{1}, \cdots, n_{2 r}\right)$ where $r \geqq 2, \phi$ is an admissible automorphism of $\mathfrak{A}(2 r), \operatorname{det}\left(D_{i} \phi x^{\epsilon_{j}}\right) \in \mathfrak{U}\left(n_{1}, \cdots, n_{2 r}\right)$ and $\phi$ stabilizes $\mathfrak{U}_{i}(2 r)$ for $1 \leqq i \leqq r$.

(10) $R(2 r+1) \cap W\left(n_{1}, \cdots, n_{2 r+1}\right)$ where $r \geqq 1, p>2$.

II. Simplicity of Lie algebras of Cartan type. Since the infinite Lie algebras of Cartan type possess filtrations satisfying conditions (1)-(6) it is not surprising that Lie algebras of Cartan type possess 
filtrations with similar properties. We use these properties to prove the simplicity of Lie algebras of Cartan type.

Definition 2. A Lie algebra $L$ is said to be strongly filtered with respect to $M$ if $L$ satisfies (1)-(5), if $L_{2} \neq(0)$ and if $M$ is a subspace of $L_{1}$ such that $L_{i} \subseteq\left[L, L_{i+1}\right]+L_{i+1}+M$ for all $i \geqq 0$.

LEMMA 2. If $L$ is a finite dimensional Lie algebra which is strongly filtered with respect to $M$ and $M^{(n)}=(0)$ then $L^{(n)}$ is simple.

TheOREM 1. Any Lie algebra of Cartan type is simple.

In view of Lemma 2 to prove Theorem 1 it is sufficient to show that each of the algebras $K$ in (7)-(10) is strongly filtered with respect to a subspace $M$ such that $M^{(2)}=(0)$. This is done separately for each of the four cases.

\section{Identification of known algebras.}

THEOREM 2. If $\Phi$ is an algebraically closed field of characteristic $p>3$ then every known finite dimensional nonclassical simple Lie algebra over $\Phi$ is of Cartan type.

We prove this by a case by case analysis of the known algebras (those listed in [14, pp. 105-110]). In the course of the proof we obtain the following complete classification of the generalized Witt algebras [13].

COROLlary. If $\Phi$ is an algebraically closed field of characteristic $p>0$ then any generalized Witt algebra over $\Phi$ is isomorphic to some $W\left(n_{1}, \cdots, n_{m}\right)$. If $W\left(n_{1}, \cdots, n_{m}\right) \cong W\left(r_{1}, \cdots, r_{s}\right)$ then $m=s$ and $n_{i}=r_{\sigma(i)}$ for $1 \leqq i \leqq m$ where $\sigma$ is a permutation of $1, \cdots, m$.

IV. New simple Lie algebras. Computation shows that if $n+3 \not \equiv 0$ $(\bmod p)$ and $n=\sum n_{i}$ then $R(2 r+1) \cap W\left(n_{1}, \cdots, n_{2 r+1}\right)$ is a simple Lie algebra of dimension $p^{n}$. If $p>3$ its derivation algebra has dimension $p^{n}+n-(2 r+1)$. By comparing these dimensions with those for the known simple Lie algebras we prove

TheOREM 3. If $p>3, n+3 \not \equiv 0(\bmod p), n>m \geqq 3$ where $m$ is an odd integer and $m \neq p^{s}+s$ for any integer $s$ then $R(m) \cap W\left(n_{1}, \cdots, n_{m}\right)$ is a new simple Lie algebra.

\section{REFERENCES}

1. A. A. Albert and M. S. Frank, Simple Lie algebras of characteristic p, Univ. e Politec. Torino Rend. Sem. Mat. 14 (1954/55), 117-139.

2. R. Block, On torsion-free abelian groups and Lie algebras, Proc. Amer. Math. Soc. 9 (1958), 613-620. 
3. - New simple Lie algebras of prime characteristic, Trans. Amer. Math. Soc. 89 (1958), 421-449.

4. E. Cartan, Les groupes de transformations continus, infinis, simples, Oeuvres completes. Vol. 2, Partie II, Gauthier-Villars, Paris, 1953, pp. 857-925.

5. M. S. Frank, $A$ new class of simple Lie algebras, Proc. Nat. Acad. Sci. U.S.A. 40 (1954), 713-719.

6. - Two new classes of simple Lie algebras, Trans. Amer. Math. Soc. 112 (1964), 456-482.

7. S. A. Jennings and R. Ree, On a family of Lie algebras of characteristic $p$, Trans. Amer. Math. Soc. 84 (1957), 192-207.

8. N. Jacobson, Restricted Lie algebras of characteristic p. II, Duke Math. J. 10 (1943), 107-121.

9. I. Kaplansky, Seminar on simple Lie algebras, Bull. Amer. Math. Soc. 60 (1954), $470-471$.

10. S. Kobayashi and T. Nagano, On filtered Lie algebras and geometric structures. II, J. Math. Mech. 14 (1965), 679-706.

11. A. I. Kostrikin and I. R. Shafarevitch, Cartan pseudogroups and Lie p-algebras, Dokl. Akad. Nauk SSSR 168 (1966), 740-742 = Soviet Math. Dokl. 7 (1966), 715-718.

12. W. H. Mills and G. B. Seligman, Lie algebras of classical type, J. Math. Mech. 6 (1957), 519-548. 546.

13. R. Ree, On generalized Witt algebras, Trans. Amer. Math. Soc. 83 (1956), 510-

14. G. B. Seligman, Modular Lie algebras, Ergebnisse der Mathematik und ihrer Grenzgebiete, vol. 40, Springer-Verlag, Berlin, 1967.

15. I. M. Singer and S. Sternberg, The infinite groups of Lie and Cartan. I. The transitive groups, J. Analyse Math 15 (1965), 1-114.

16. S. Sternberg, Lectures on differential geometry, Prentice-Hall, Englewood Cliffs, N. J., 1964.

Yale University, New Haven, Connecticut 06520 